\title{
NUMERICAL SIMULATION OF THE EFFECT OF CHEMICAL OSMOSIS ON THE VALUE OF THE JUMPS OF POLLUTION IN THE GEOCHEMICAL BARRIER
}

\author{
Oksana V. Ulianchuk-Martyniuk ${ }^{1}$, Olha R. Michuta ${ }^{2} \S$ \\ ${ }^{1,2}$ National University of Water \\ and Environmental Engineering \\ Rivne, UKRAINE
}

\begin{abstract}
The mathematical model of the propagation of chemical substances in the soil that contains a thin clay geobarrier was investigated. The function of the geobarrier as an integral element of the soil base of waste storage facilities is the limitation of the spread of harmful substances outside the facility. The developed mathematical model takes into account: 1) the ideality properties of the barrier material; 2) the phenomenon of chemical osmosis; 3) the conjugation conditions account for the dependence of the filtration coefficient, the degree of ideality, the diffusion coefficient on the concentration of the chemicals. The necessity to consider such dependencies is substantiated by the review of scientific publications with the corresponding data of field experiments. The numerical solution of the corresponding non-linear boundary value problem was found by the finite element method. The numerical experiments show the significance of considering chemical osmosis in the prediction calculations of the spread of harmful chemicals through geobarriers.
\end{abstract}

AMS Subject Classification: 34B60, 35Q35, 65M60

Key Words: chemical waste; geobarrier; chemical osmosis; semipermeability; conjugation condition; mathematical model; finite element method

\section{Introduction}

When liquid inorganic chemical contaminants enter into clay soil, osmotic phe-

Received: July 21, 2020

(C) 2020 Academic Publications

${ }^{\S}$ Correspondence author 
nomena may appear in the porous medium. This is due to the specific properties of clay soils and the possibility of their action as semi-permeable membranes. These are membranes that have a rather high permeability not for all but only for some substances, in particular for solvents. Osmosis is a one-sided diffusion through a semi-permeable membrane that separates the solution from a pure solvent or a solution of lower concentration. This phenomenon is explained by the gravitation of the system to thermodynamic equilibrium and the leveling of the concentration of the solutions on both sides of the membrane. It is characterized by osmotic pressure that is equal to the excessive external pressure which must be applied from the side of the solution to stop osmosis [10, 19]. A detailed analysis (164 primary sources) of the research of osmotic effects in porous media including both theoretical and experimental developments was performed in [17]. The problems and effects of chemical osmosis, electro-osmosis and thermal osmosis were revealed. As noted in [5], it is the soils with low permeability, which include clay, that can operate as semi-permeable membranes that generate osmotic flow.

Although much investigation of osmosis for clay soils has been performed, both in terms of on-site experimental research and predictive mathematical modeling $[28,29]$, some aspects have not been taken into account so far. One of these is osmotic phenomena under the conjugation conditions for thin geosynthetic barriers in the soil base of the storage facilities for household and industrial wastes. As practice shows, clay and geosynthetic barriers are widely used in the construction of such facilities. A detailed review (a total of 128 primary sources) of the reports on the use of geosynthetic thin coatings (inclusions) in waste storage facilities was performed in [9]. The authors note that such inclusions of geosynthetic clay materials began to be widely used since 1970s. Their primary function is to restrict the spread of harmful substances from waste storage facilities to the environment. The use of artificial geosynthetic bentonite geobarriers in the waste storages was reviewed in [22]. It is noted that industrial GCL (geosynthetic clay liners) are currently manufactured as panels of 4.2-5.3 meter width, 30-61 meter length, and 15 to $30 \mathrm{~mm}$ thickness. According to scientific sources, GCLs as finished panels were used for the first time in 1986.

A review of current technologies and research on the use of improved bentonites as geobarriers for inorganic waste storage was the objective of [23]. They focused on three characteristics: the filtration coefficient, the effective diffusion coefficient and the membrane ideality coefficient $\omega$ (the chemico-osmotic efficiency coefficient of the membrane). The necessity to improve the properties of the geosynthetic bentonite geobarriers arises from certain solutions of chemical 
industry waste to which bentonites are still not stable and are weak as functioning protective and restraining barriers. Processes of mechanical and chemical consolidation of clay materials that exhibit the properties of semipermeable membranes are investigated in [30]. They used a modification of the Darcy law

$$
v_{u}+v_{\pi}=-k \frac{\partial h}{\partial z}+\omega \frac{k}{\gamma} \frac{\partial \pi}{\partial z}=-k \frac{\partial h}{\partial z}+\omega \frac{R T}{M} \frac{k}{\gamma} \frac{\partial c}{\partial z}
$$

where $\omega$ is the chemico-osmotic efficiency coefficient of the membrane; $M$ is the molar mass of the solute; $k$ is the filtration coefficient; $\pi$ is the osmotic pressure; $R$ is the universal gas constant; $T$ is the absolute temperature; $c$ is the concentration of chemicals in the pore fluid. It was also noted that according to $[7]$

$$
k=k_{0}\left(\frac{n}{n_{0}}\right)^{3},
$$

where $n$ is porosity; $k_{0}, n_{0}$ are the initial values of the filtration coefficient and of porosity. It should be noted that the issues of chemical and thermal consolidation of soils taking into account osmotic phenomena were also studied in $[28,29]$. However, the heterogeneity of the environment and the presence of thin semipermeable inclusions were not considered. For a flow of a chemical solution we have

$$
q_{c}=(1-\omega) v_{u} c+v_{\pi} c-n D^{*} \frac{\partial c}{\partial z},
$$

where $D^{*}$ is the effective diffusion coefficient including "the tortuosity of porous media flow paths". It was shown in [13] for semipermeable clay materials that

$$
D^{*}=\tau(1-\omega) D_{0}
$$

where $D_{0}$ is the diffusion coefficient for a solution in the porous liquid; $\tau$ is the tortuosity factor and $0<\tau<1$. According to [12]

$$
\tau=n^{m}
$$

where $m$ is an empirical parameter. Also, numerical experiments were performed and certain numerical parameters of the model were determined in [30].

Chemical osmosis in [19] is defined as the possibility of the movement of water (solvent) under the influence of salt concentration difference. In this case, the generalized Darcy law takes the form

$$
q=-k \frac{\partial h}{\partial x}+\omega k \frac{\partial \pi_{h}}{\partial x} .
$$


Here $k$ is the filtration coefficient; $h$ is the heads; $\pi_{h}$ is the osmotic heads, or $\pi_{h}=\frac{\pi}{\rho g}=\frac{\pi}{\gamma}$, where $\pi$ is the osmotic pressure. Osmotic pressure is defined as $[3]$

$$
\pi=\frac{R T}{v_{w}} \ln \left(a_{w}\right),
$$

where $R$ is the universal gas constant; $T$ is the temperature; $v_{w}$ is the molar volume of water; $a_{w}$ is water activity (dimensionless value).

It was experimentally established in [24] that the effective diffusion coefficient of $\mathrm{KCl}$ solution in geosynthetic bentonite geobarriers non-linearly depends on the value of the input concentration $C_{0}$ (Table 1 ). The dependence of the ideality coefficient on $C_{0}$ is also presented. Obtained experimental results confirm the possibility of significant mutual effect of the concentration of chemical substances dissolved in pore water and physico-chemical parameters of the porous medium.

\begin{tabular}{|l|l|l|}
\hline$C_{0}(m M)$ & $D \times 10^{-5}\left(\mathrm{~m}^{2} /\right.$ day $)$ & $\omega$ \\
\hline 20 & 0.76896 & 0.37 \\
\hline 35 & 0.93312 & 0.24 \\
\hline 50 & 1.01088 & 0.18 \\
\hline 100 & 1.08864 & 0.11 \\
\hline 200 & 1.08864 & 0.11 \\
\hline 400 & 1.22688 & 0 \\
\hline
\end{tabular}

Table 1. Dependence of the effective diffusion coefficient and the ideality coefficient of geosynthetic geobarrier on the input concentration of $\mathrm{KCl}$ [24]

The ratio of the filtration coefficient $k_{0}$ of the geosynthetic barrier in the presence of $\mathrm{KCl}$ solution of concentration $C_{0}$ in the pores to the filtration coefficient $K_{0}$ in pure water was investigated in [14]. The results shown in Table 2 testify to the need to take into account even modest concentrations of chemical solutions in pores through the change in filtration characteristics of geobarriers. After all, the filtration coefficient $k_{0}$ increases 1.65 times faster than $K_{0}$ with increasing $\mathrm{KCl}$ concentration to $C_{0}=50 \mathrm{mM}$.

\begin{tabular}{|l|l|}
\hline \multicolumn{1}{|c|}{$C_{0}(m M)$} & $k_{0} / K_{0}$ \\
\hline 0 & 1 \\
\hline 10 & 1.2 \\
\hline 20 & 1.35 \\
\hline 30 & 1.5 \\
\hline 40 & 1.6 \\
\hline 50 & 1.65 \\
\hline
\end{tabular}


Table 2. Dependence of the filtration coefficient ratio of geosynthetic geobarrier on the input concentration of $\mathrm{KCl}[14]$

The above research overview outlines existing developments and still outstanding tasks regarding the spread of pollutants in environments with clay geobarriers. Particularly, the well-known modifications of the Darcy law can take into account the ideality properties (coefficient $\omega$ ) as well as the phenomena of osmosis for geobarriers. The results of many reports point to the importance of such tasks in terms of ecology and environmental protection. However, the results of field experiments show that the filtration coefficient $k$ and the ideality coefficient $\omega$ non-linearly depend both on the concentration of chemicals $c$ and the humidity $\theta$. Therefore, the corresponding mathematical model of the distribution of pollutants in an unsaturated medium with thin clay inclusions will be described by a boundary value problem with non-linear dependence of coefficients on unknown functions. Moreover, in [6] the conjugation conditions were modified for the presence of osmosis phenomena and for non-linear dependence of the parameters of thin inclusions on the determinant functions of processes (concentration of chemical solutions and humidity). However, no studies of the effect of such dependences on the processes themselves were performed. That is the objective of this article.

The process of the spread of dissolved substances by diffusion through a soil mass acting as a limiting geobarrier for the contaminants was considered in [4]. Assuming linear pressure distribution in the pore fluid, the generally non-linear diffusion equation was reduced to a linear one. Due to this, an analytical solution to the respective boundary value problem was found. Generally, numerical methods have to be applied in the case of non-linear problems. The investigation of the effect of the non-linear dependences $k=k(c, \theta)$ and $\omega=\omega(c)$ as well as the osmosis phenomenon itself on the spread of pollutants in the environment with geobarriers requires solving a non-linear boundary value problem for the systems of differential equations of parabolic type. The complexity of the problem is determined also by the possibility of occurrence of discontinuities in solutions.

The application of the finite element method (FEM) to models of hydraulic fracturing of the formation in a porous medium is described in [15]. The gap and the jump in the fields of the head is "smoothed out" (reduces to a continuous case) through the introduction of the concept of "weak rupture". Algorithmic and computational aspects of the application of FEM to mathematical models of hydraulic fracturing in porous media with possible discontinuities in the solutions are discussed in [20]. Discretization of the two-dimensional problem utilized finite elements of two types, classic triangular finite elements in the 
sub-regions without cracks, and finite elements of zero "thickness" with triple nodes in the split itself (one node on one side of the crack, another in the center of the crack that belongs to a one-dimensional finite element, the third one on the other side of the crack). In the assumption of small deformations, the thickness of the crack during the discretization is disregarded. The idea of triple nodes is the expansion of the idea of dual nodes in the places of possible discontinuities in the solution $[25,26]$.

A new approach (according to the authors) to the numerical study of the process of the propagation of cracks in heterogeneous media which may consist of discrete elements is proposed in [2]. Among the numerical methods, the attention is focused on FEM, an overview of its existing modifications and development of new ones. Thus, the scientists consider FEM one of the key methods for finding approximate solutions of boundary value problems in heterogeneous media with inclusions. This is due to the possibility of finding the most generalized approximate solutions. Given the relative simplicity of implementation, in this paper we use the approach to numerical solution of this type of problems that is described in $[25,26]$.

\section{A mathematical model of the problem}

Consider a one-dimensional case. The interconnected process of the variation of the heads and the concentration of a chemical substance in a pore solution of an unsaturated heterogeneous soil mass in the presence of chemical osmosis is described by the following boundary-value problem:

$$
\begin{gathered}
\beta(h) \frac{\partial h}{\partial t}=\frac{\partial}{\partial x}\left(k(h, c) \frac{\partial h}{\partial x}-k(h, c)-k_{c}(h, c) \frac{\partial c}{\partial x}\right), \\
x \in \Omega_{1} \cup \Omega_{2}, t>0 ; \\
\left.h(x, t)\right|_{x=0}=\bar{h}_{0}(t), t \geq 0 ; \\
\left.u(x, t)\right|_{x=l}=\left.\left(-k(h, c) \frac{\partial h}{\partial x}+k(h, c)-k_{c}(h, c) \frac{\partial c}{\partial x}\right)\right|_{x=l}=0, t \geq 0 ; \\
h(x, 0)=h_{0}(x), x \in[0 ; \xi] \cup[\xi ; l] ; \\
\theta(h) \frac{\partial c}{\partial t}=\frac{\partial}{\partial x}\left(\theta(h) D(h, c) \frac{\partial c}{\partial x}\right)-u \frac{\partial c}{\partial x}, x \in \Omega_{1} \cup \Omega_{2}, t>0 ; \\
\left.c(x, t)\right|_{x=0}=\bar{c}_{0}(t), t \geq 0 ;
\end{gathered}
$$




$$
\begin{gathered}
\left.q_{c}(x, t)\right|_{x=l}=-\left.\theta(h) D(h, c) \frac{\partial c}{\partial x}\right|_{x=l}=0, t \geq 0 ; \\
c(x, 0)=c_{0}(x), x \in[0 ; \xi] \cup[\xi ; l] ; \\
\left.u^{ \pm}\right|_{x=\xi}=-\frac{[h]}{\int_{0}^{d} \frac{d x}{k^{\gamma}(h, c)}}+\frac{d}{\int_{0}^{d} \frac{d x}{k^{\gamma}(h, c)}}+\frac{1}{\int_{0}^{d} \frac{d x}{k^{\gamma}(h, c)} \int_{0}^{d} \frac{k_{c}^{\gamma}(h, c)}{k^{\gamma}(h, c)} \frac{\partial c}{\partial x} d x ;} \\
\left.q_{c}^{ \pm}\right|_{x=\xi}=-(1-\omega(\theta, c)) \frac{[c]}{\int_{0}^{d} \frac{d x}{\theta(h) D^{\gamma}(h, c)}} .
\end{gathered}
$$

Eqs. (1), (5) neglect the internal sources and drains of moisture and chemicals including mass transfer processes. Here $d$ is the thickness of the thin inclusion; $\Omega_{1}=(0 ; \xi), \Omega_{2}=(\xi ; l), 0<\xi<l ; \bar{c}_{0}(t), \bar{h}_{0}(t), c_{0}(x), h_{0}(x)$ are known functions; $k_{c}$ and $k_{c}^{\gamma}$ are the osmosis coefficients of the main soil and the fine inclusion, respectively, with $k_{c}=\omega(h, c) \frac{R T}{M} \frac{k(h, c)}{\gamma}, k_{c}^{\gamma}=\omega^{\gamma}(h, c) \frac{R T}{M} \frac{k^{\gamma}(h, c)}{\gamma}$, and $\omega(h, c), \omega^{\gamma}(h, c)$ are the chemico-osmotic efficiency coefficients of the membrane. Functions $h_{0}(x), c_{0}(x)$ must be continuous at each of the segments $[0 ; \xi],[\xi ; l]$. Eq. (1) is the moisture transfer equation $[1,27]$. According to van Genuchten's model [27],

$$
\theta(h)=\theta_{\min }+\frac{\theta_{\max }-\theta_{\min }}{\left(1+(-\alpha h)^{n}\right)^{m}}, k(h)=k_{0} \sqrt{s}\left(1-\left(1-s^{\frac{1}{m}}\right)^{m}\right)^{2},
$$

where $\theta \in\left[\theta_{\min } ; \theta_{\max }\right], \theta_{\min }>0, \theta_{\max } \in\left(\theta_{\min } ; 1\right], n>1, m=1-\frac{1}{n} ; s=$ $\frac{\theta-\theta_{\min }}{\theta_{\max }-\theta_{\min }}$ is relative humidity; $k_{0}$ is the filtration coefficient (for full saturation with $s=1$ and $h=0)$. Further,

$$
\beta(h)=\frac{d \theta}{d h}=\alpha n m\left(\theta_{\max }-\theta_{\min }\right)\left(1+(-\alpha h)^{n}\right)^{-m-1}(-\alpha h)^{n-1} .
$$

Also, the filtration coefficient $k_{0}$ significantly depends on the concentration of chemicals dissolved in the pore fluid $[11,14]$.

Conditions (9), (10) are the modified conjugation conditions in the case of the dependence of the parameters of fine inclusion and its ideality degree on physico-chemical factors as well as the consideration of chemical osmosis [6]. Here $q_{c}$ is the flow of chemicals through the fine inclusion; $[c]=c^{+}-c^{-}$is the value of the gap in the concentration of chemicals at the inclusion. Since we place the source of moisture and chemical contaminants on top $(x=0)$, then the ideality coefficient is assumed to depend on the concentration of chemicals and the humidity at $x=\xi-0$. 


\section{Solving the problem in the area with the inclusion by the finite element method}

Similarly to $[25,26]$, we introduce a number of definitions and spaces. Let $H_{0}$ be a space of vector-valued functions $\left\{s_{1}(x) ; s_{2}(x)\right\}$ each of the components of which at each of the intervals $(0 ; \xi),(\xi ; l)$ belong to the Sobolev space $W_{2}^{1}(\Omega)$ and acquire zero values at the ends of the segment $[0, l]$ where the boundary conditions of the first kind are set for the functions $h(x, t), c(x, t)$, respectively. Let $H$ be a space of functions $\left\{v_{1}(x, t) ; v_{2}(x, t)\right\}$ each component of which is square-integrable along with the first derivatives $\frac{\partial v_{i}}{\partial t}, \frac{\partial v_{i}}{\partial x}, i=1,2$ at each of the intervals $(0 ; \xi),(\xi ; l), \forall t \in(0 ; T]$, and satisfy the same boundary conditions of the first kind as the functions $h(x, t), c(x, t)$, respectively. Here $T>0$.

Let $\left\{s_{1}(x) ; s_{2}(x)\right\} \in H_{0}$. Multiplying Eq. (1) and the initial condition (4) by $s_{1}(x)$, integrating them over the segment $[0, l]$ and taking into account the conjugation condition (9), we obtain

$$
\begin{array}{r}
\int_{0}^{l} \beta(h) \frac{\partial h}{\partial t} s_{1}(x) d x+\int_{0}^{l} k(h, c) \frac{\partial h}{\partial x} \frac{d s_{1}(x)}{d x} d x+\frac{[h]\left[s_{1}\right]}{\int_{0}^{d} \frac{d x}{k^{\gamma}(h, c)}} \\
-\int_{0}^{l} k_{c}(h, c) \frac{\partial c}{\partial x} \frac{d s_{1}(x)}{d x} d x-\frac{[c]\left[s_{1}\right]}{d} \frac{\int_{0}^{d} \frac{k_{c}^{\gamma}(h, c)}{k^{\gamma}(h, c)} d x}{\int_{0}^{d} \frac{d x}{k^{\gamma}(h, c)}} \\
=\int_{0}^{l} k(h, c) \frac{d s_{1}(x)}{d x} d x+\frac{d\left[s_{1}\right]}{\int_{0}^{d} \frac{d x}{k^{\gamma}(h, c)}}, \forall t \in(0 ; T] ; \\
\int_{0}^{l} h(x, 0) s_{1}(x) d x=\int_{0}^{l} h_{0}(x) s_{1}(x) d x, \forall t \in(0 ; T] .
\end{array}
$$

Similarly, from Eqs. (5) and (8)

$$
\int_{0}^{l} \theta(h) \frac{\partial c}{\partial t} s_{2}(x) d x+\int_{0}^{l} \theta(h) D(h, c) \frac{\partial c}{\partial x} \frac{d s_{2}(x)}{d x} d x+\int_{0}^{l} u \frac{\partial c}{\partial x} s_{2}(x) d x
$$




$$
\begin{gathered}
+\left(1-\omega\left(\theta^{-}, c^{-}\right)\right) \frac{[c]\left[s_{2}\right]}{\int_{0}^{d} \frac{d x}{\theta(h) D^{\gamma}(h, c)}}=0, \quad \forall t \in(0 ; T] ; \\
\quad \int_{0}^{l} c(x, 0) s_{2}(x) d x=\int_{0}^{l} c_{0}(x) s_{2}(x) d x, \forall t \in(0 ; T] .
\end{gathered}
$$

Definition 1. A function $\{h(x, t) ; c(x, t)\} \in H$ which for any $\left\{s_{1}(x) ; s_{2}(x)\right\} \in$ $H_{0}$ satisfies the integral relations (11)-(14) is called a generalized solution of the boundary value problem (1)-(10).

An approximate generalized solution of the boundary value problem (1)-(10) is sought in the form

$$
h(x, t)=\sum_{i=1}^{N} a_{i}(t) \phi_{i 1}(x), c(x, t)=\sum_{i=1}^{N} b_{i}(t) \phi_{i 2}(x),
$$

where $\left\{\phi_{i 1}(x) ; \phi_{i 2}(x)\right\}, i=\overline{2, N}$ are basis vector-functions of finite-dimensional subspace $M_{0} \in H_{0}$ and functions $\phi_{11}(x), \phi_{12}(x)$ are used for the approximation of non-uniform boundary conditions of the first kind (2) and (6).

Further, from the weak formulation (11)-(14) of the problem (1)-(10), taking into account (15), we obtain the Cauchy problem

$$
\begin{gathered}
\mathbf{M}_{11}(\mathbf{A}, \mathbf{B}) \cdot \frac{d \mathbf{A}}{d t}+\mathbf{L}_{11}(\mathbf{A}, \mathbf{B}) \cdot \mathbf{A}(t)+\mathbf{L}_{12}(\mathbf{A}, \mathbf{B}) \cdot \mathbf{B}(t)=\mathbf{F}_{11}(\mathbf{A}, \mathbf{B}), \\
\tilde{\mathbf{M}}_{1} \cdot \mathbf{A}^{(0)}=\tilde{\mathbf{F}}_{1}, \\
\mathbf{M}_{22}(\mathbf{A}, \mathbf{B}) \cdot \frac{d \mathbf{B}}{d t}+\mathbf{L}_{22}(\mathbf{A}, \mathbf{B}) \cdot \mathbf{B}(t)=\mathbf{F}_{22}(\mathbf{A}, \mathbf{B}), \\
\tilde{\mathbf{M}}_{2} \cdot \mathbf{B}^{(0)}=\tilde{\mathbf{F}}_{2},
\end{gathered}
$$

where $\mathbf{A}=\left(a_{i}(t)\right)_{i=1}^{N}, \mathbf{A}^{(0)}=\left(a_{i}(0)\right)_{i=1}^{N}, \mathbf{M}_{k k}=\left(m_{i j}^{(k k)}\right)_{i, j=1}^{N}, \mathbf{L}_{k k}=\left(l_{i j}^{(k k)}\right)_{i, j=1}^{N}$, $\mathbf{F}_{k k}=\left(f_{i}^{(k k)}\right)_{i=1}^{N}, \tilde{\mathbf{F}}_{k}=\left(\tilde{f}_{i}^{(k)}\right)_{i=1}^{N}, \tilde{\mathbf{M}}_{k}=\left(\tilde{m}_{i j}^{(k)}\right)_{i, j=1}^{N}, k=1,2, \mathbf{L}_{12}=\left(l_{i j}^{(12)}\right)_{i, j=1}^{N} ;$

$$
\begin{gathered}
m_{i j}^{(11)}=\int_{0}^{l} \beta(\hat{h}) \phi_{i 1} \phi_{j 1} d x, \tilde{m}_{i j}^{(1)}=\int_{0}^{l} \phi_{i 1} \phi_{j 1} d x, \tilde{f}_{i}^{(1)}=\int_{0}^{l} h_{0} \phi_{i 1} d x, \\
l_{i j}^{(11)}=\int_{0}^{l} k(\hat{h}, \hat{c}) \frac{d \phi_{i 1}}{d x} \frac{d \phi_{j 1}}{d x} d x+\frac{\left[\phi_{i 1}\right]\left[\phi_{j 1}\right]}{\int_{0}^{d} \frac{d x}{k^{\gamma}(\hat{h}, \hat{c})}},
\end{gathered}
$$




$$
\begin{gathered}
l_{i j}^{(12)}=\frac{\left[\phi_{i 1}\right]\left[\phi_{j 1}\right]}{d} \int_{0}^{l} k_{c}(\hat{h}, \hat{c}) d x+\frac{\left[\phi_{i 1}\right]\left[\phi_{j 1}\right]}{d \int_{0}^{d} \frac{d x}{k^{\gamma}(\hat{h}, \hat{c})}} \int_{0}^{d} \frac{k_{c}^{\gamma}(\hat{h}, \hat{c})}{k^{\gamma}(\hat{h}, \hat{c})} d x, \\
f_{i}^{(11)}=\int_{0}^{l} k(\hat{h}, \hat{c}) \frac{d \phi_{i 1}(x)}{d x} d x+\frac{d\left[\phi_{i 1}\right]}{\int_{0}^{d} \frac{d x}{k^{\gamma}(\hat{h}, \hat{c})}}, \\
m_{i j}^{(22)}=\int_{0}^{l} \theta(\hat{h}) \phi_{i 2} \phi_{j 2} d x, \tilde{m}_{i j}^{(2)}=\int_{0}^{l} \phi_{i 2} \phi_{j 2} d x, \tilde{f}_{i}^{(2)}=\int_{0}^{l} c_{0} \phi_{i 2} d x, f_{i}^{(22)}=0, \\
l_{i j}^{l}=\int_{0}^{l} \theta(h) D(\hat{h}, \hat{c}) \frac{d \phi_{j 2}}{d x} \frac{d \phi_{i 2}}{d x} d x+\int_{0}^{l} u \frac{d \phi_{j 2}}{d x} \phi_{i 2} d x+ \\
+\left(1-\omega\left(\theta^{-}, c^{-}\right)\right) \frac{\left[\phi_{i 2}\right]\left[\phi_{j 2}\right]}{\int_{0}^{d} \frac{d x}{\theta(h) D^{\gamma}(\hat{h}, \hat{c})}}
\end{gathered}
$$

\section{Some practical aspects of the finite-element solving of the problem (1)-(10)}

In practice, approximate finding of $h(x, t)$ and $c(x, t)$ utilizes the same net of finite elements. Then $\phi_{i 1}(x), \phi_{i 2}(x)$, therefore to avoid double indexation, we denote these basis functions $\phi_{i}(x), i=\overline{1, N}$. Similar to $[25,26]$, we cover $[0 ; \xi] \cup[\xi ; l]$ with a finite-element grid. There are two finite elements in this case $\left[x_{m-1} ; x_{m}\right] \in[0 ; \xi]$ and $\left[x_{m+1} ; x_{m+2}\right] \in[\xi ; l]$ such that $x_{m}=x_{m+1}=\xi$ and $x_{m} \in[0 ; \xi], x_{m+1} \in[\xi ; l]$. Basis functions $\phi_{m}(x)$ and $\phi_{m+1}(x)$ are discontinuous at $x=\xi$, i.e.

$$
\begin{gathered}
\left.\phi_{m}(x)\right|_{x=\xi-0}=1,\left.\phi_{m}(x)\right|_{x=\xi+0}=0, \\
\left.\phi_{m+1}(x)\right|_{x=\xi-0}=0,\left.\phi_{m+1}(x)\right|_{x=\xi+0}=1 .
\end{gathered}
$$

To simplify, time discretization is performed on a uniform grid with step $\tau$. The number of steps in time is set as $M$. To find out $\mathbf{B}^{(p+1)}$, we perform 
discretization of the system of non-linear differential equations (18) over time using a completely implicit linearized difference scheme [16]

$$
\begin{gathered}
\mathbf{M}_{22}\left(\mathbf{A}^{(p)}, \mathbf{B}^{(p)}\right) \cdot \frac{\mathbf{B}^{(p+1)}-\mathbf{B}^{(p)}}{\tau}+\mathbf{L}_{22}\left(\mathbf{A}^{(p)}, \mathbf{B}^{(p)}\right) \cdot \mathbf{B}^{(p+1)} \\
=\mathbf{F}_{22}\left(\mathbf{A}^{(p)}, \mathbf{B}^{(p)}\right), p=1,2, \ldots
\end{gathered}
$$

where $\mathbf{A}^{(p)}=\mathbf{A}\left(t_{p}\right), \mathbf{B}^{(p)}=\mathbf{B}\left(t_{p}\right), t_{p}=p \tau$. After the formation of the linear system

$$
\mathbf{G} \cdot \mathbf{B}^{(\mathbf{p}+\mathbf{1})}=\mathbf{F}
$$

where

$$
\begin{gathered}
\mathbf{G}=\frac{1}{\tau} \mathbf{M}_{11}\left(\mathbf{A}^{(p)}, \mathbf{B}^{(p)}\right)+\mathbf{L}_{11}\left(\mathbf{A}^{(p)}, \mathbf{B}^{(p)}\right), \\
\mathbf{F}=\frac{1}{\tau} \mathbf{M}_{11}\left(\mathbf{A}^{(p)}, \mathbf{B}^{(p)}\right) \cdot \mathbf{A}^{(p)}+\mathbf{F}_{11}\left(\mathbf{A}^{(p)}, \mathbf{B}^{(p)}\right),
\end{gathered}
$$

we must take into account the principal boundary condition - the boundary condition of the first kind (2). This is accomplished by the following two steps: 1) all elements of the first equation of the linear system, including the free member, are nullified; 2) the first element of the first equation is set equal to 1 , and the free member is set equal to $c\left(t_{p+1}\right)$. The integrals in the formation of $\mathbf{G}$ and $\mathbf{F}$ are found by numerical integration. Similarly, $\mathbf{A}^{(p+1)}$ is found from (16) using obtained values $\mathbf{B}^{(p+1)}$.

\section{Results of numerical experiments}

Soil parameters for the numerical experiments are taken from the Hydrus-1D program [8]. As the main soil, loam with $k_{0}=0.028 \mathrm{~m} /$ day, $\theta_{\min }=0.1$, $\theta_{\max }=0.38, n=1.23, \alpha=2.7 \mathrm{day}^{-1}$ were considered. As a soil of thin inclusion, clay with the following parameters was used: $k_{0}^{\gamma}=0.0048 \mathrm{~m} / \mathrm{day}$, $\theta_{\min }=0.07, \theta_{\max }=0.36, n=1.09, \alpha=0.5 \mathrm{day}^{-1}$. The values of $R=$ $8.31446261815 \mathrm{~J} / \mathrm{mol} \cdot \mathrm{K}, T=293.15 \mathrm{~K}\left(20^{0} \mathrm{C}\right), M=74.5513 \cdot 10-3 \mathrm{~kg} / \mathrm{mol}$, $\gamma=104 \mathrm{~N} / \mathrm{m}^{3}$ were used. It was assumed that the main soil does not have osmotic properties, and that its ideality coefficient equals zero.

For the model problem, the layer of soil of $l=3 m$ thickness was considered. The depth of the inclusion was $\xi=1.5 m$, and its thickness $d=0.2 \mathrm{~m}$. The step of variable $x$ was $0.005 m$, the time step $\tau=1 d a y$. The starting distribution of heads was $h_{0}(x)=-20 \mathrm{~m}$. The original concentration of $\mathrm{KCl}$ in the chemical solution was $c_{0}(x)=8 \mathrm{mM}$. The functions in the boundary conditions of the 
first kind at the surface of the soil were $\bar{h}_{0}(t)=0 \mathrm{~m}, \bar{c}_{0}(t)=400 \mathrm{mM}$. The results of the numerical experiments are presented in Tables 3-5.

\begin{tabular}{|l|c|c|c|c|c|c|}
\hline \multicolumn{1}{|c|}{ Time moment } & $h^{-}$ & $h^{+}$ & {$[h]$} & $c^{-}$ & $c^{+}$ & {$[c]$} \\
\hline$t=180$ days & 0.3685 & 0.3319 & -0.0366 & 41.82 & 47.92 & -0.9 \\
\hline$t=360$ days & 0.3757 & 0.3556 & -0.0201 & 94.80 & 65.91 & -28.89 \\
\hline$t=720$ days & 0.3780 & 0.3674 & -0.0106 & 198.80 & 151.12 & -47.68 \\
\hline$t=1080$ days & 0.3800 & 0.3800 & 0 & 220.94 & 175.13 & -45.81 \\
\hline
\end{tabular}

Table 3. No semi-permeable properties of the clay inclusion $\left(\omega^{\gamma}=0\right)$

\begin{tabular}{|l|l|l|l|l|l|l|}
\hline \multicolumn{1}{|c|}{ Time moment } & \multicolumn{1}{c|}{$h^{-}$} & \multicolumn{1}{c|}{$h^{+}$} & \multicolumn{1}{c|}{$[h]$} & $c^{-}$ & $c^{+}$ & {$[c]$} \\
\hline$t=180$ days & 0.3753 & 0.2651 & -0.1102 & 38.78 & 20.65 & -18.13 \\
\hline$t=360$ days & 0.3800 & 0.2668 & -0.1132 & 69.92 & 17.73 & -52.19 \\
\hline$t=720$ days & 0.3800 & 0.2591 & -0.1209 & 116.21 & 23.24 & -92.97 \\
\hline$t=1080$ days & 0.3800 & 0.3800 & 0 & 147.04 & 29.18 & -117.86 \\
\hline
\end{tabular}

Table 4. Consideration of osmosis and the ideality degree dependent on the $\mathrm{KCl}$ concentration

\begin{tabular}{|l|l|l|l|l|l|l|}
\hline \multicolumn{1}{|c|}{ Time moment } & \multicolumn{1}{c|}{$h^{-}$} & \multicolumn{1}{c|}{$h^{+}$} & \multicolumn{1}{c|}{$[h]$} & $c^{-}$ & $c^{+}$ & {$[c]$} \\
\hline$t=180$ days & 0.3731 & 0.2896 & -0.0835 & 38.84 & 20.83 & -18.01 \\
\hline$t=360$ days & 0.3761 & 0.3532 & -0.0229 & 99.45 & 21.22 & -78.23 \\
\hline$t=720$ days & 0.3777 & 0.3653 & -0.0124 & 219.74 & 37.38 & -182.36 \\
\hline$t=1080$ days & 0.3800 & 0.3800 & 0 & 271.07 & 48.18 & -222.89 \\
\hline
\end{tabular}

Table 5. Consideration only of the $\mathrm{KCl}$ concentration-dependent ideality degree and neglecting osmosis

The salt diffusion coefficient in the main soil was taken as [1]

$$
D_{1}|u|+\theta D_{2} \tau_{2}
$$

where $D_{1}$ is the hydrodynamic dispersion coefficient; $D_{2}$ is the molecular diffusion coefficient. According to $[8,18]$

$$
\tau_{2}=\frac{\theta^{\frac{7}{3}}}{\theta_{\max }^{2}} .
$$


For numerical experiments, aqueous $\mathrm{KCl}$ solution was considered as a chemical substance, with $D_{2}=1.721952 \cdot 10^{-5} \mathrm{~m}^{2} /$ day [21]. The hydrodynamic dispersion coefficient in this model problem was equal to the molecular diffusion coefficient. The $\mathrm{KCl}$ diffusion coefficient in a thin inclusion depended on the salt concentration according to the data of Table 1.

The results presented in Tables 3-5 show that both the semi-permeability and the inclusion of osmotic phenomena are essential in the predictive calculations. For instance, in the absence of the ideality of the geobarrier, the pollution level beyond the geobarrier after three years is already $175 \mathrm{mM}$ and differs from the level before the geobarrier only by $45 \mathrm{~m} M$. When disregarding osmosis (Table 5), the pollution level beyond the geobarrier is $48 \mathrm{mM}$ which is much less than the results of the experiment according to Table 3. However, this is almost twice as high as for the case of the simultaneous consideration of the ideality degree and chemical osmosis (Table 4). Given that the process is long-term, the projected amount of contaminants transferred beyond the boundaries of the waste storage into ground water can vary considerably depending on the conditions for solving the problem. Therefore, it is important to take into full account the data of field observations and experiments in the model.

\section{Conclusions}

Thus, in this paper the mathematical model of the propagation of chemical substances in the soil that contains a thin clay geobarrier was investigated. The developed mathematical model takes into account: 1) the ideality properties of the barrier material; 2) the phenomenon of chemical osmosis; 3 ) the conjugation conditions account for the dependence of the filtration coefficient, the degree of ideality, the diffusion coefficient on the concentration of the chemi-cals. The numerical solution of the corresponding non-linear boundary value problem was found by the finite element method. The results of numerical experiments show that both the semi-permeability and the inclusion of osmotic phenomena are essential in the predictive calculations. For instance, in the absence of the ideality of the geobarrier, the pollution level beyond the geobarrier after three years differs from the level before the geobarrier only by $26 \%$. When disregarding osmosis, the pollution level beyond the geobarrier differs from the level before the geobarrier by $463 \%$. For the case of the simultaneous consideration of the ideality degree and chemical osmosis, the difference is about $400 \%$. The numerical experments show the significance of considering chemical osmosis in the prediction calculations of the spread of harmful chemicals through geobarriers. 


\section{References}

[1] J. Bear, Dynamics of Fluid in Porous Media, Elsevier, New York (1972).

[2] M.F. Benedetto, A. Caggiano, G. Etse, Virtual elements and zero thickness interface-based approach for fracture analysis of heterogeneous materials, Computer Methods in Applied Mechanics and Engineering, 338 (2018), 41-67.

[3] E. Bresler, Anion exclusion and coupling effects in nonsteady transport through unsaturated soils: I. Theory, Soil Sci. Soc. Am. J., 5, No 37 (1973), 663-669.

[4] R. Chen, Y. Ge, Z. Chen, J. Liu, Y. Zhao, Z. Li, Analitical solution for one-dimensional contaminant diffusion through unsaturated soils beneath geomembrane, J. of Hydrology, 568 (2019), 260-274.

[5] X. Chen, S.F. Thornton, W. Pao, Mathematical model of coupled dual chemical osmosis based on mixture-coupling theory, Int. J. Engineering Science, 129 (2018), 145-155.

[6] Yu. Chui, P. Martyniuk, M. Kuzlo, O. Ulianchuk-Martyniuk, The conditions of conjugation in the tasks of moisture transfer on a thin clay inclusion taking into account salt salutions and temperature, J. of Theoret. and Appl. Mech., 49, No 1 (2019), 28-38.

[7] R.D. Hart, C.S. John, Formulation of a fully coupled thermal-mechanicalfluid flow model for non-linear geologic systems, Int. J. Rock Mech. Min. Sci. Geomech. Abstr., 3, No 23 (1986), 213-224.

[8] D. Jacques, J. Šimůnek, D. Mallants, M.T. van Genuchten, The HPx software for multicomponent reactive transport during variably-saturated flow: Recent developments and applications, J. Hydrol. Hydromech., 66, No 2 (2018), 211-226.

[9] Kong De-Jun, Wu Huai-Na, Chai Jin-Chun, Arulrajah Arul, State-Of-TheArt Review of Geosynthetic Clay Liners, Sustainability, 9, No 11 (2017), 2110.

[10] E.M. Kramer, D.R. Myers, Five popular misconceptions about osmosis, American J. of Physics, 80, No 8(2012), 694-699. 
[11] M.T. Kuzlo, P.M. Martyniuk, Filtration of saline solutions in soil environments, Academic J., Ser.: Industrial Machine Building, Civil Engin., 49, No 2 (2017), 76-81.

[12] J.G. Liu, H.T. Wang, Y.F. Nie, Fractal model for predicting effective diffusion coefficient of solute in porous media, Adv. Water Sci., 15, No 4 (2004), 458-462.

[13] M.A. Malusis, J.B. Kang, C.D. Shackelford, Restricted salt diffusion in a geosynthetic clay liner, Int. Wireless Communications and Mobile Computing Conference (2014), 134-139.

[14] M.A. Malusis, C.D. Shackelford, Chemico-osmotic efficiency of a geosynthetic clay liner, J. Geotech. Geoenviron. Eng., 128, No 2 (2002), 97-106.

[15] O.L. Manzoli, P.R. Cleto, M. Sanchez, L.J.N. Guimaraes, M.A. Maedo, On the use of high aspect ratio finite elements to model hydraulic fracturing in deformable porous media, Comput. Methods Appl. Mech. Engrg., 350 (2019), 57-80.

[16] P.M. Martyniuk, O.R. Michuta, O.V. Ulianchuk-Martyniuk, M.T. Kuzlo, Numerical investigation of pressure head jump values on a thin inclusion in one-dimensional non-linear soil mousture transport problem, International Journal of Applied Mathematics, 31, No 4 (2018), 649-660; DOI:10.12732/ijam.v31i4.10.

[17] I. Medved, R. Cerny, Osmosis in porous media: A review of recent studies, Microporous and Mesoporous Materials, 170 (2013), 299-317.

[18] R.J. Millington, J.M. Quirk, Permeability of porous solids, Trans. Faraday Soc., 57 (1961), 1200-1207.

[19] C.E. Neuzil, M. Person, Reexamining ultrafiltration and solute transport in groundwater, Water Resources Research, 53, No 6 (2017), 4922-4941.

[20] V.P. Nguyen, H. Lian, T. Rabczuk, S. Bordas, Modelling hydraulic fractures in porous media using flow cohesive interface elements, Engineering Geology, 225 (2017), 68-82.

[21] A.A. Ravdel, A.M. Ponomaryova, I. Fedorov (Eds.), Short Handbook of Physico-Chemical Values, Saint-Petersburg (2003). 
[22] J. Scalia, C.A. Bareither, C.D. Shackelford, Advancing the use of geosynthetic clay liners as barriers, Geotechnical Engin. J. of the SEAGS \& AGSSEA, 49, No 4 (2018), 100-114.

[23] J. Scalia, G.L. Bohnhoff, C.D. Shackelford, C.H. Benson, K.M. SampleLord, M.A. Malusis, W.J. Likos, Enhanced bentonites for containment of inorganic waste leachates by GCLs, Geosynthetics Internat., 25, No 4 (2018), 392-411.

[24] C.D. Shachelford, A. Meier, K. Sample-Lord, Limiting membrane and diffusion behavior of a geosynthetic clay liner, Geotextiles and Geomembranes, 44, No 5 (2016), 707-718.

[25] I.V. Sergienko, V.S. Deineka, V.V. Skopetskii, High-accuracy discretization algorithms for problems with discontinuous solutions, Cybernetics and Systems Analysis, 34, No 4 (1998), 489-505.

[26] V.V. Skopetskii, V.S. Deineka, L.I. Sklepovaya, T.P. Savosh, Calculating unsteady seepage in a pressure gradient in the presence of thin weakly permeable inclusions, J. of Mathematical Sciences, 72, No 2 (1994), 2992-2997.

[27] M.T. van Genuchten, A closed-form equation for predicting the hydraulic conductivity of unsaturated soils, Soil Sci. Soc. Amer. J., 44, No 5 (1980), 892-898.

[28] A.P. Vlasyuk, P.M. Martynyuk, Numerical solution of three-dimensional problems of filtration consolidation with regard for the influence of technogenic factors by the method of radial basis functions, J. of Mathematical Sciences, 171, No 5 (2010), 632-648.

[29] A.P. Vlasyuk, P.M. Martynyuk, O.R. Fursovych, Numerical solution of a one-dimensional problem of filtration consolidation of saline soils in a nonisothermal regime, J. of Mathematical Sciences, 160, No 4 (2009), 525-535.

[30] Zh. Zhang, Sh.A. Masum, H.R. Thomas, L. Han, Modeling fully coupled hydraulic-mechanical-chemical processes in a natural clay liner under mechanical and chemico-osmotic consolidation, Environmental Sci. and Pollution Research, 25 (2018), 36173-36183. 